11,12

\title{
Критические температуры модели локальных бозонов на квадратной решетке в приближении Бете
}

\author{
(C) Е.Л. Спевак, Ю.Д. Панов, А.С. Москвин \\ Уральский федеральный университет, \\ Екатеринбург, Россия \\ E-mail: spevak155@gmail.com
}

Поступила в Редакцию 9 апреля 2021 г.

В окончательной редакции 9 апреля 2021 г.

Принята к публикации 19 апреля 2021 г.

\begin{abstract}
Рассмотрен учет ближних корреляций для двумерной модели локальных бозонов на квадратной решетке в рамках приближения Бете для кластеров из двух и четырех узлов. Получены явные уравнения для определения критических температур зарядового и сверхтекучего упорядочения и рассмотрены их решения для различных отношений параметра межцентровых заряд-зарядовых корреляций и интеграла переноса. Показано, что учет ближних корреляций для температур зарядового упорядочения приводит к появлению критической концентрации бозонов, ограничивающей область существования решений типа зарядового упорядочения. Для сверхтекучего упорядочения при учете ближних корреляций происходит редукция критической температуры вплоть до нулевых значений при половинном заполнении. Построена фазовая диаграмма модели локальных бозонов с учетом расслоения фаз в рамках построения Максвелла, и показано, что учет ближних корреляций в приближении Бете количественно приближает вид фазовой диаграммы к результатам квантового метода Монте-Карло.
\end{abstract}

Ключевые слова: локальные бозоны, фазовая диаграмма, неравенство Боголюбова, приближение Бете.

DOI: $10.21883 /$ FTT.2021.09.51265.08H

\section{1. Введение}

Модели решеточных бозонов активно привлекаются для описания систем, демонстрирующих нетривиальную фазовую диаграмму с конкурирующими или смешанными состояниями. Первоначально модель локальных (hard-core) бозонов была предложена для объяснения особенностей фазовой диаграммы сверхтекучего ${ }^{4} \mathrm{He}[1,2]$, и в настоящее время различные варианты этой модели используются для описания свойств большого числа физических систем, включающих высокотемпературные сверхпроводники $[3,4]$, ультрахолодные атомы в оптических решетках [5], экситоны в нанотрубках [6] и магнитные изоляторы во внешнем поле [7]. Интерес к этим моделям в последние годы вырос после обнаружения сосуществования зарядового упорядочения и сверхпроводимости в купратах $[8,9,10]$.

Гамильтониан локальных заряженных бозонов имеет вид $[1,3]$ :

$$
\mathscr{H}=-t \sum_{\langle i, j\rangle}\left(b_{i}^{+} b_{j}+b_{j}^{+} b_{i}\right)+V \sum_{\langle i, j\rangle} n_{i} n_{j}-\mu \sum_{i} n_{i},
$$

где $b^{+}(b)$ - операторы рождения (уничтожения) локальных бозонов с коммутационными соотношениями Бозе-типа для разных узлов $\left[b_{i}, b_{j}^{+}\right]=0, i \neq j$, и Фермитипа на одном узле: $\left\{b_{i}, b_{i}^{+}\right\}=1, n_{i}=b_{i}^{+} b_{i}$ - оператор числа локальных бозонов на узле, $t$ и $V$ - интеграл переноса и параметр заряд-зарядовых корреляций между ближайшими соседями, $\mu-$ химический потенциал, необходимый для учета условия постоянства концентрации бозонов: $n N=\sum_{i}\left\langle n_{i}\right\rangle$, где $N-$ полное число узлов. Далее мы рассматриваем локальные бозоны на плоской квадратной решетке.

Диаграмма основного состояния системы локальных бозонов в приближении среднего поля (ПСП) хорошо известна $[3,11]$ и показана на рис. $1, a$ в переменных $(n, V / t)$. Левее гейзенберговской точки $V / t=2$ основным состоянием при всех $n$ является сверхтекучая жидкость (superlfuid, SF), правее при $n=1 / 2$ реализуется зарядовое упорядочение (charge ordering, $\mathrm{CO}$ ), при $|n-1 / 2|>\eta^{*} \eta^{*}=\frac{1}{2} \sqrt{(V / t-2) /(V / t+2)}$, реализуется SF-фаза, а при $0<|n-1 / 2|<\eta^{*}$ основное состояние представляет собой сверхтекучее твердое тело (supersolid, SS) - фазу, в которой отличны от нуля одновременно зарядовый и сверхтекучий параметры порядка. Условия для существования SS-фазы, ее устойчивость к фазовому расслоению (phase separation, PS) на макроскопические домены SF- и CO-фаз были и остаются предметом дискуссии и многочисленных исследований. Численные расчеты методом квантового Монте-Карло показывают стабильность SS-фазы в моделях с фрустрирующими взаимодействиями: на треугольной решетке [12-15], либо на квадратной с учетом взаимодействия со вторыми соседями [16-19]. Также устойчивая SS-фаза найдена в различных вариантах модели Бозе-Хаббарда [5]. Для модели (1) в ПСП в основном состоянии энергии и области существования 

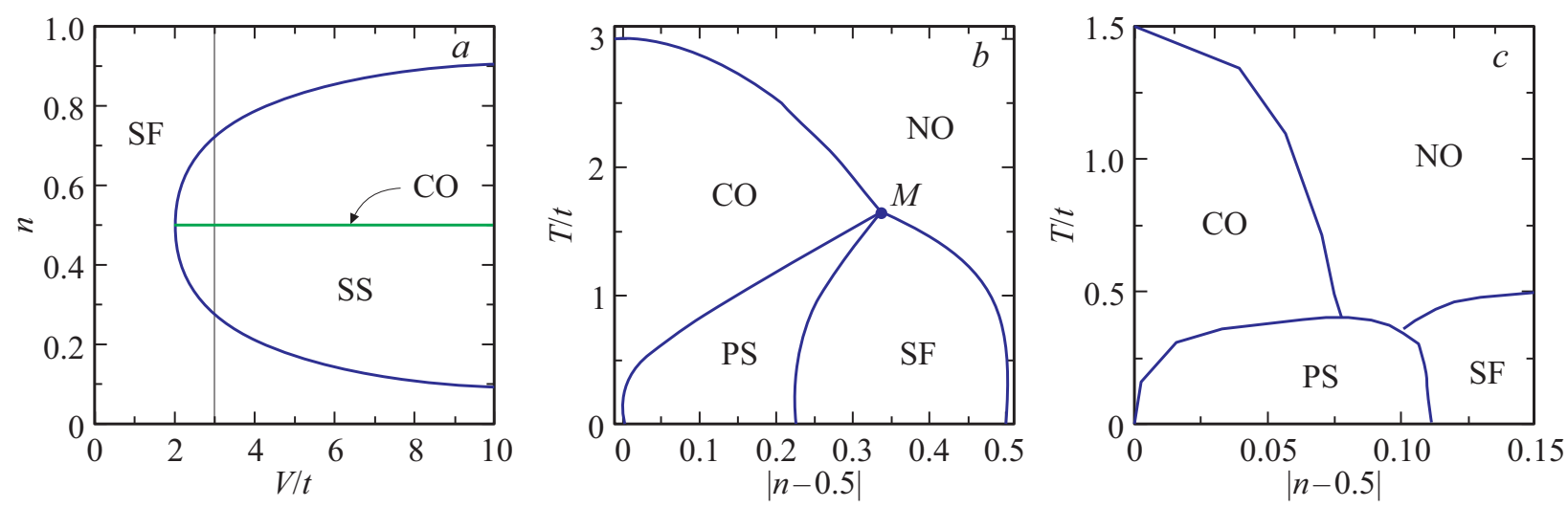

Pис. 1. (а) Диаграмма основного состояния модели (1) в приближении среднего поля. SF - сверхтекучая жидкость, SS сверхтекучее твердое тело, СО - зарядовое упорядочение. (b) Фазовая диаграмма в приближении среднего поля при $V / t=3$ (соответствует серой вертикальной линии на панели $(a)$ ). PS - фазовое расслоение на макроскопические домены SF- и CO-фаз, NO - неупорядоченная фаза. (c) Фазовая диаграмма, полученная методом квантового Монте-Карло при $V / t=3$ (по данным работы [22]).

PS- и SS-фазы совпадают, а при конечной температуре SS-фаза метастабильна [20,21]: ее энергия всегда выше PS-состояния. Фазовая $(n, T)$-диаграмма модели (1) в ПСП [11] показана на рис. $1, b$ для $V / t=3$. Ее интересная особенность состоит в наличии CO-фазы при $n \neq 1 / 2$, которая при понижении температуры переходит в PS-состояние. Область PS начинается с трикритической точки $M$. Полученная в [22] методом квантового Монте-Карло фазовая диаграмма модели (1) для $V / t=3$ (см. рис. $1, c$ ) разительно отличается от результатов среднего поля: масштаб критических температур существенно ниже, значительно меньше область концентраций, где реализуется СО-фаза, а также вместо трикритической точки возникает область концентраций, при которых происходит переход из неупорядоченной фазы (non-ordered, NO) в PS-фазу.

Значительная часть исследований моделей решеточных бозонов посвящена расчетам методом квантового Монте-Карло. Аналитически различные характеристики системы локальных бозонов исследовались, кроме метода среднего поля, с учетом дальних корреляций в рамках метода спиновых волн [3,23-27] и разных вариантах метода функций Грина $[3,8,29]$. В настоящей работе рассмотрено влияние учета ближних корреляций в рамках приближения Бете. Для кластеров из двух и четырех узлов найдены критические температуры модели (1), и рассмотрена эволюция фазовых диаграмм в переменных $(n, T)$ в зависимости от этих приближений.

Статья организована следующим образом. В разделе 2 записаны основные соотношения для приближения Бете в модели локальных бозонов на двумерной квадратной решетке. В разделе 3 исследовано поведение критических температур для различных соотношений $V / t$, а также построены фазовые $(n, T)$-диаграммы с учетом фазового расслоения. Мы сравниваем эти результаты с ПСП и квантового метода Монте-Карло. Последний раздел посвящен выводам.

\section{2. Приближение Бете для кластеров из двух и четырех узлов}

Система локальных бозонов эквивалентна квантовому магнетику с постоянной намагниченностью - системе псевдоспинов $s=1 / 2$ во внешнем поле, направленном вдоль $z$-оси [1], описываемой гамильтонианом

$$
\begin{aligned}
\mathscr{H}= & -t \sum_{\langle i, j\rangle}\left(s_{i}^{+} s_{j}^{-}+s_{i}^{-} s_{j}^{+}\right)+V \sum_{\langle i, j\rangle} s_{i}^{z} s_{j}^{z} \\
& -h \sum_{i} s_{i}^{z}+E_{0},
\end{aligned}
$$

где $s_{i}^{-}=b_{i}^{+}, s_{i}^{+}=b_{i}, s_{i}^{z}=\frac{1}{2}-n_{i}, h=2 V-\mu, E_{0}=N \varepsilon_{0}$ $=N(V-\mu) / 2$.

Для оценки большого потенциала $\Omega(\mathscr{H})$ мы используем неравенство Боголюбова: $\Omega(\mathscr{H}) \leq \Omega=\Omega\left(\mathscr{H}_{0}\right)$ $+\left\langle\mathscr{H}-\mathscr{H}_{0}\right\rangle$, где гамильтониан нулевого приближения $\mathscr{H}_{0}$ имеет вид суммы гамильтонианов $\mathscr{H}_{c}$ для невзаимодействующих кластеров Бете из $c$ узлов, покрывающих всю решетку: $\mathscr{H}_{0}=\Sigma \mathscr{H}_{c}$. Варианты используемых нами кластеров показаны на рис. 2. Определим на квадратной решетке две подрешетки $\alpha=A, B$, и запишем выраже-

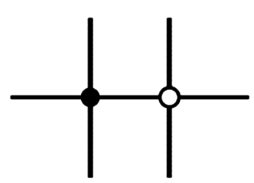

MFA

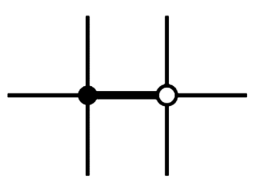

2

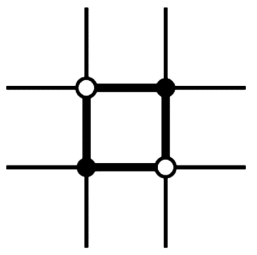

4
Рис. 2. Вид кластеров Бете, используемых при вычислении гамильтониана (3). ПСП соответствует кластер из невзаимодействующих узлов. Черными кружками обозначены узлы подрешетки $A$, белыми - узлы подрешетки $B$. 
ние для $\mathscr{H}_{c}$ в виде

$$
\begin{aligned}
\mathscr{H}_{c}= & -t \sum_{\langle i, j\rangle}^{c}\left(s_{i}^{+} s_{j}^{-}+s_{i}^{-} s_{j}^{+}\right)+V \sum_{\langle i, j\rangle}^{c} s_{i}^{z} s_{j}^{z} \\
& -\mathbf{g}_{f} \sum_{i=1}^{c} \mathbf{s}_{i}-\mathbf{g}_{a} \sum_{i=1}^{c}(-1)^{\alpha_{i}} \mathbf{s}_{i} .
\end{aligned}
$$

Здесь суммирование производится по узлам кластера, $(-1)^{A}=1,(-1)^{B}=-1, \mathbf{g}_{f, a}-$ молекулярные поля, являющиеся вариационными параметрами и учитывающие взаимодействие кластера Бете с окружением. Формально, ПСП соответствует гамильтониан (3), содержащий только слагаемые с молекулярными полями.

Изотропный характер переноса в плоскости $(x, y)$ позволяет ограничиться только $x$ - и $z$-компонентами молекулярных полей. Вычислив статистическую сумму для кластера $Z_{c}=\operatorname{Tr} \exp \left(-\beta \mathscr{H}_{c}\right)$, где $\beta=1 / k_{\mathrm{B}} T$ (и далее мы полагаем $\left.k_{\mathrm{B}}=1\right)$, запишем выражения через молекулярные поля для величины отклонения концентрации бозонов от половинного заполнения, $\eta=1 / 2-n$, и различных параметров порядка (ПП):

$$
\begin{aligned}
& \eta=\frac{1}{c \beta} \frac{\partial \ln Z_{c}}{\partial g_{f}^{z}}, \quad \xi=\frac{1}{c \beta} \frac{\partial \ln Z_{c}}{\partial g_{a}^{z}}, \\
& \rho=\frac{1}{c \beta} \frac{\partial \ln Z_{c}}{\partial g_{f}^{x}}, \quad \chi=\frac{1}{c \beta} \frac{\partial \ln Z_{c}}{\partial g_{a}^{x}} .
\end{aligned}
$$

Здесь $\xi$ и $\rho-$ зарядовый и сверхтекучий ПП, $\chi-$ асиметрия сверхтекучего ПП.

Оценка большого потенциала в расчете на один узел решетки, $\omega=\Omega / N$, имеет вид

$$
\begin{aligned}
\omega= & -\frac{1}{c \beta} \ln Z_{c}+a V\left(\eta^{2}-\xi^{2}\right)-2 a t\left(\rho^{2}-\chi^{2}\right) \\
& +\eta g_{f}^{z}+\xi g_{a}^{z}+\rho g_{f}^{x}+\chi g_{a}^{x}-h \eta+\varepsilon_{0},
\end{aligned}
$$

где $a=2$ для ПСП, $a=3 / 2$ для кластера из двух узлов и $a=1$ для кластера из четырех узлов. Минимизируя $\omega$ по молекулярным полям, получим уравнения для ПП:

$$
\begin{gathered}
2 a V \eta=-g_{f}^{z}+h, \quad 2 a V \xi=g_{a}^{z}, \\
4 a t \rho=g_{f}^{x}, \quad 4 a t \chi=-g_{a}^{x} .
\end{gathered}
$$

Критические температуры найдем из условия потери устойчивости минимума $\omega$ для NO-фазы относительно вариации по соответствующему молекулярному полю. Для температуры зарядового упорядочения, $T_{\mathrm{CO}}$, получим уравнение

$$
\left.2 a V\left(\frac{\partial \xi}{\partial g_{a}^{z}}\right)\right|_{0}=1,
$$

а для температуры сверхтекучего перехода, $T_{\mathrm{SF}}$, уравнение имеет вид

$$
\left.4 a t\left(\frac{\partial \rho}{\partial g_{f}^{x}}\right)\right|_{0}=1 .
$$

В уравнениях (7) и (8) производная вычисляется при $g_{a}^{z}=0, g_{f}^{x}=0, g_{a}^{x}=0$. Для получения зависимости от концентрации бозонов необходимо эти уравнения решать совместно с уравнением (4) для $\eta$. Также отметим, что аналогичное уравнение для критической температуры перехода беспорядок-порядок, связанное с вариацией $g_{a}^{x}$, не имеет решения.

Область PS на фазовой диаграмме в переменных $(n, T)$ можно найти используя построение Максвелла [30-32]. Решая уравнения (6) отдельно для СО-фазы и для SF-фазы, найдем параметр $\eta^{*}$ при котором значения большого потенциала в этих фазах равны, $\omega_{\mathrm{CO}}\left(\eta^{*}\right)=\omega_{\mathrm{SF}}\left(\eta^{*}\right)$, и из выражения (4) для $\eta$ определим граничные концентрации области $\mathrm{PS}, \eta_{\mathrm{CO}}\left(\eta^{*}\right)$ и $\eta_{\mathrm{SF}}\left(\eta^{*}\right)$.

\section{3. Результаты}

Уравнения (7) и (8) в ПСП приводят к хорошо известным выражениям [11] для критических температур

$$
\frac{T_{\mathrm{CO}}}{V}=1-4 \eta^{2}, \quad \frac{T_{\mathrm{SF}}}{2 t}=4 \eta\left(\ln \frac{1+2 \eta}{1-2 \eta}\right)^{-1} .
$$

В случае кластера Бете из 2 узлов уравнение (7) для $T_{\mathrm{CO}}$ принимает вид

$$
\frac{\operatorname{ch}(\beta t)+2 g}{2 \operatorname{sh}(\beta t)}=\frac{3 V}{4 t}\left(1-4 \eta^{2}\right),
$$

где

$$
g=\frac{1}{2} \sqrt{\left(1-4 \eta^{2}\right) e^{-\beta V}+4 \eta^{2} \operatorname{ch}^{2}(\beta t)} .
$$

Уравнение (8) для $T_{\mathrm{SF}}$ с учетом выражения (4) для кластера Бете из 2 узлов можно привести к виду

$$
\begin{aligned}
& x^{2}-\left(\frac{V}{2}+t\right)^{2} \\
& =6 t\left(\left(\frac{V}{2}+t\right) \frac{2 \eta^{2} \operatorname{ch}(\beta t)-2 e^{\beta t}\left(\frac{1}{4}-\eta^{2}\right)+g}{\operatorname{ch}(\beta t)+2 g}+\eta x\right),
\end{aligned}
$$

где

$$
x=\beta^{-1} \ln ((2 \eta \operatorname{ch}(\beta t)+2 g) /(1-2 \eta))+V / 2 .
$$

Для кластера Бете из четырех узлов явный вид уравнений для критических температур становится более громоздким. Уравнение (7) для ТСО можно записать в следующем виде:

$$
\begin{aligned}
& Z_{0}^{-1}\left[\left(2 \beta V+\frac{V}{t} \operatorname{sh}(2 \beta t)\right) \operatorname{ch}(\beta y)\right. \\
& \left.+e^{\frac{1}{2} \beta V} \frac{V^{2}}{2 t^{2}}\left(\operatorname{ch}(\beta d)+\frac{V^{2} / 2+8 t^{2}}{V d} \operatorname{sh}(\beta d)-e^{\frac{1}{2} \beta V}\right)\right]=1,
\end{aligned}
$$

где $d=\sqrt{V^{2} / 4+8 t^{2}}$,

$$
\begin{aligned}
Z_{0}= & 3+e^{\beta V}+2 e^{\frac{1}{2} \beta V} \operatorname{ch}(\beta d)+2 e^{-\beta V} \operatorname{ch}(2 \beta y) \\
& +4(1+\operatorname{ch}(2 \beta t)) \operatorname{ch}(\beta y) .
\end{aligned}
$$

Здесь $Z_{0}$ имеет смысл статистической суммы кластера Бете в NO-фазе. Уравнение (12) необходимо решать 

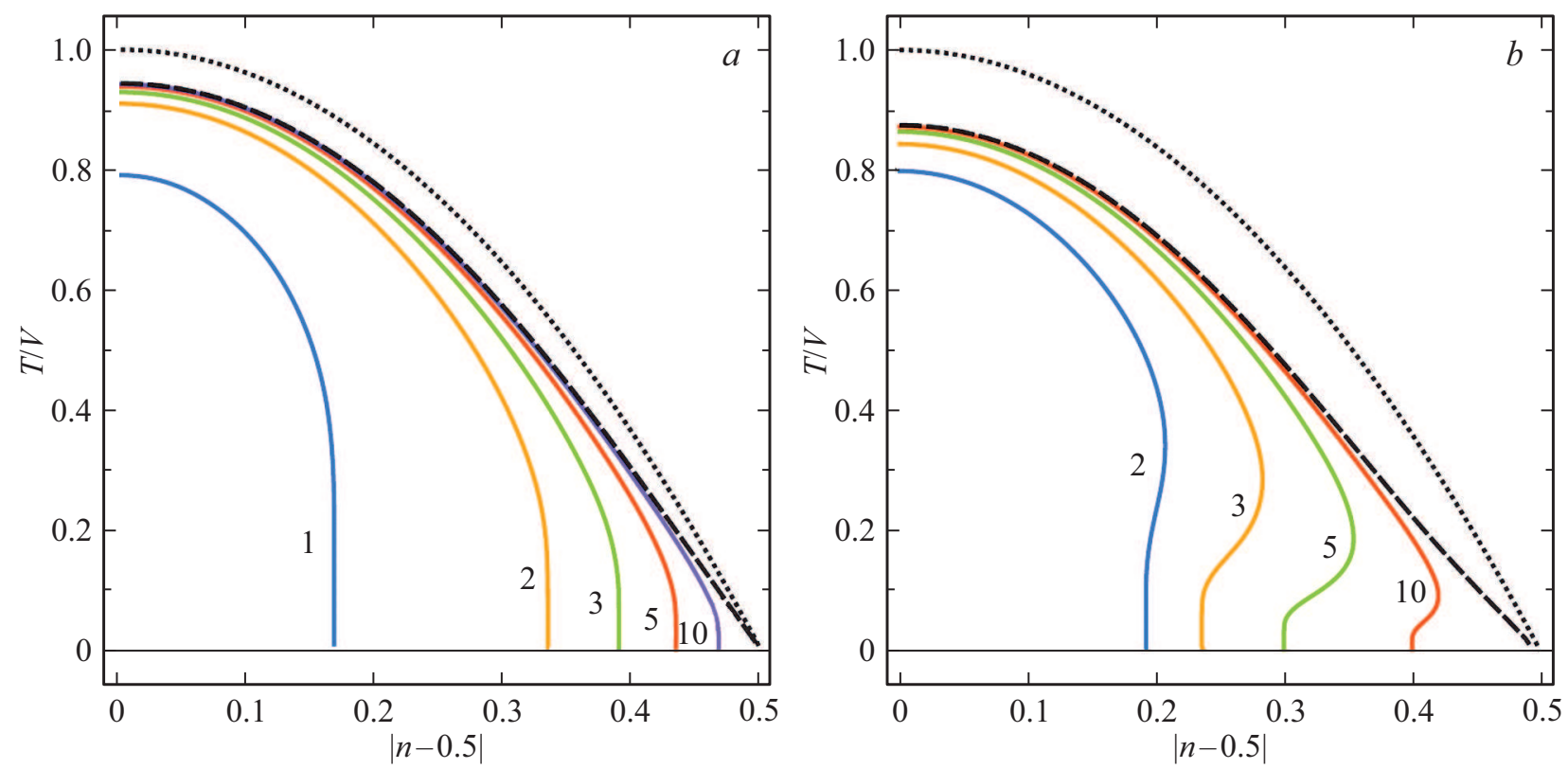

Рис. 3. Приведенные критические температуры $\left.T_{\mathrm{CO}} \quad a\right)$ для двухузельного приближения Бете, уравнение (10), $b)$ для четырехузельного приближения Бете, уравнение (12). Числа рядом с кривыми соответствуют значениям $V / t$. Штриховая линия показывает предельный случай, $V / t \rightarrow \infty$, пунктирная линия - приведенную критическую температуру зарядового упорядочения в ПСП, уравнение (9).

совместно с уравнением (4) для $\eta$, которое в данном случае можно записать в виде

$$
Z_{0}^{-1} \operatorname{sh}(\beta y)\left(1+\operatorname{ch}(2 \beta t)+2 e^{-\beta V} \operatorname{ch}(\beta y)\right)=\eta .
$$

Наконец, уравнение (8) для поиска $T_{\mathrm{SF}}$ можно явно представить в следующем виде:

$$
\begin{aligned}
2 t Z_{0}^{-1}\left[\frac{2}{y}\right. & \operatorname{sh}(\beta y)-\alpha e^{\frac{1}{2} \beta V}\left(b_{1} \operatorname{ch}(\beta d)+b_{2} \operatorname{sh}(\beta d)\right) \\
& +c e^{\beta V}-2 e^{-\beta V}(c \operatorname{ch}(2 \beta y)+s \operatorname{sh}(2 \beta y)) \\
& +e^{2 \beta t}\left(b_{3} c \operatorname{ch}(\beta y)+b_{4} s \operatorname{sh}(\beta y)\right) \\
& \left.-e^{-2 \beta t}(c \operatorname{ch}(\beta y)+s \operatorname{sh}(\beta y))\right]=1 .
\end{aligned}
$$

Здесь введены величины $a=2 t+V, c=a /\left(a^{2}-y^{2}\right)$, $s=y /\left(a^{2}-y^{2}\right)$,

$$
\begin{aligned}
b_{0}= & y^{4}-y^{2}\left(24 t^{2}-4 t V+V^{2}\right)+4 a^{2} t^{2}, \\
b_{1}= & \left(y^{2}+4 t(V-7 t)\right) / b_{0}, \\
b_{2}= & \left(y^{2}(8 t+V)-4 t\left(40 t^{2}-5 t V+V^{2}\right)\right) / b_{0}, \\
b_{3}= & \left(y^{4}-4 a^{2} t(5 t-V)-y^{2}(V-4 t)^{2}\right) / b_{0}, \\
b_{4}= & \left(y^{2}\left(32 t^{2}+4 t V+3 V^{2}\right)\right. \\
& \left.-2 a^{2}\left(30 t^{2}-8 t V+V^{2}\right)-y^{4}\right) /\left(2 d b_{0}\right) .
\end{aligned}
$$

Уравнение (15) также необходимо решать совместно с уравнением (14).
На рис. 3 показаны решения уравнений (10) и (12) для разных отношений $V / t$. В случае ПСП приведенная критическая температура зарядового упорядочения, $T_{\mathrm{CO}} / V$, согласно уравнению (9) описывается универсальной параболической зависимостью от концентрации бозонов для всех значений параметра $t$. Учет ближних корреляций в рамках приближения Бете приводит к количественному уменьшению величины $T_{\mathrm{CO}} / V$ и к качественному изменению вида зависимостей критической температуры зарядового упорядочения от концентрации бозонов. Для конечной величины отношения $V / t$ возникает критическое значение концентрации бозонов, $\left|n_{c}-1 / 2\right|=\eta_{c}$, ограничивающее область существования критической температуры зарядового упорядочения. В случае двухузельного приближения Бете анализ уравнения (10) приводит к значению $\eta_{c}=1 / 2-t / 3 V$, что позволяет найти критическое значение отношения $V / t=2 / 3$, ниже которого решения уравнения (10) для $T_{\mathrm{CO}}$ не существует. Отметим также, что для значений $V / t$ ниже гейзенберговской точки, $V / t<2$, речь идет о решениях уравнения (10) для метастабильных состояний (для локальных минимумов большого потенциала), а при $V / t>2$ решения уравнения (10) соответствуют переходу $\mathrm{NO}-\mathrm{CO}$ только до трикритической точки. Численный анализ уравнения (12) показывает, что для четырехузельного приближения Бете решений не существует при $V / t<1$. Как видно из рис. 3, предельная при $V / t \rightarrow \infty$ зависимость для $T_{\mathrm{CO}}$ существует во всем диапазоне концентраций, но максимальное значение приведенной температуры при половинном заполнении оказывается ниже значения ПСП на 5.5\% 

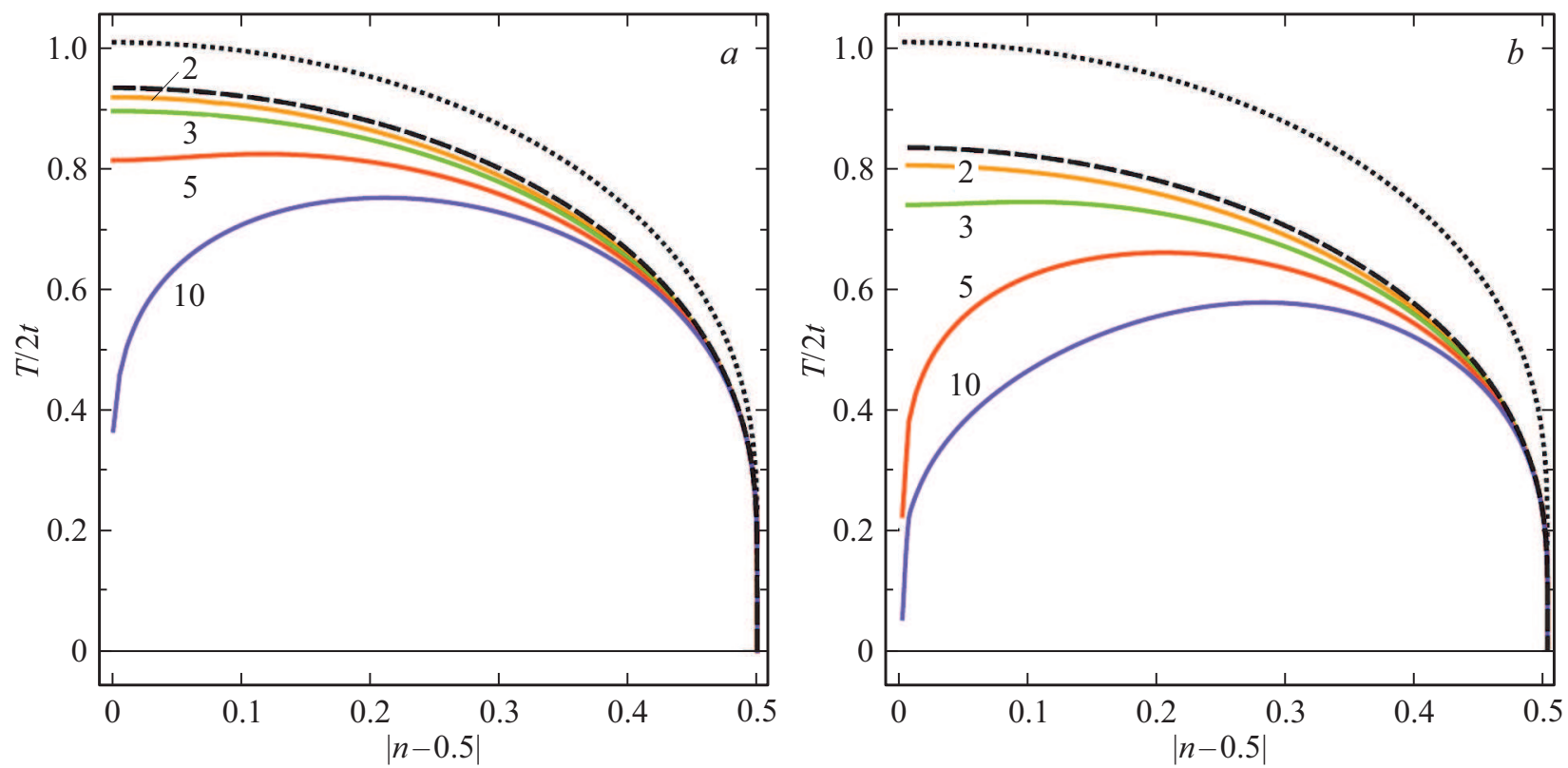

Рис. 4. Приведенные критические температуры $\left.T_{\mathrm{SF}} a\right)$ для двухузельного приближения Бете, уравнение (11), $b)$ для четырехузельного приближения Бете, уравнение (15). Числа рядом с кривыми соответствуют значениям $V / t$. Штриховая линия показывает предельный случай, $V / t=0$, пунктирная линия - приведенную критическую температуру сверхтекучего упорядочения в ПСП, уравнение (9).

для двухузельного и на $12.5 \%$ для четырехузельного приближения Бете.

Приведенные критические температуры сверхтекучего упорядочения, $T_{\mathrm{SF}} / 2 t$, являющиеся решениями уравнений (11) и (15) для разных отношений $V / t$ показаны на рис. 4. Универсальная зависимость для ПСП (9) соответствует пунктирной линии. Учет ближних корреляций в двух- и четырехузельном приближении Бете приводит к редукции величины $T_{\mathrm{SF}} / 2 t$ с ростом отношения $V / t$. Во всех случаях этот эффект более выражен для 4-узельного приближения. Качественное изменение состоит в уменьшении $T_{\mathrm{SF}}$ вплоть до нулевых значений при половинном заполнении с ростом отношения $V / t$. Предельная зависимость приведенной критической температуры при $V / t \rightarrow 0$ как и в случае ПСП имеет максимальную температуру при половинном заполнении, но величина оказывается ниже значения ПСП на $7.5 \%$ для для двухузельного и на $16.5 \%$ для для четырехузельного приближения Бете. В отличии от случая зарядового упорядочения, для $T_{\mathrm{SF}}$ решения уравнений (11) и (15) существуют во всем интервале концентраций $0<|n|<1 / 2$, но при $V / t>2$, переходу $\mathrm{NO}-\mathrm{SF}$ отвечают только концентрации правее трикритической точки.

На рис. 5 приведена фазовая диаграмма модели локальных бозонов, полученная в двух- и четырехузельном приближении Бете при $V / t=3$. Пунктирными линиями показаны для сравнения результаты ПСП [11]. Учет ближних корреляций приводит к сокращению областей для CO- и PS-фазы: уменьшается как максимальная температура, так и диапазон концентраций. Максимум температуры зарядового упорядочения при половинном заполнении уменьшается относительно ПСП приблизительно на 7\% для двухузельного приближения и на $16 \%$ для четырехузельного приближения. Для кван-

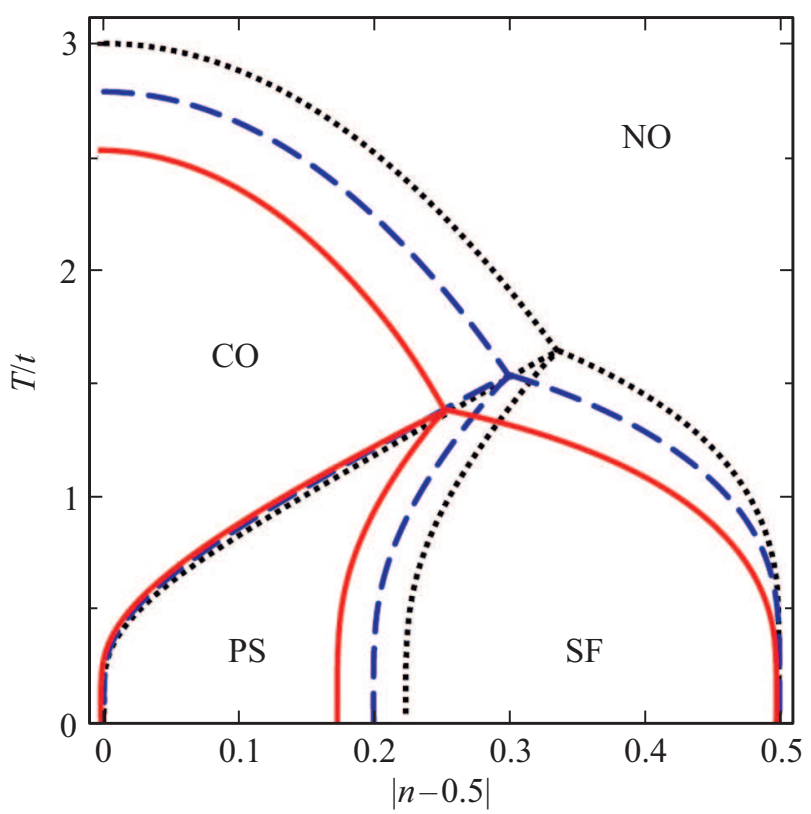

Рис. 5. Фазовая диаграмма модели локальных бозонов при $V / t=3$. Пунктирная линия соответствует ПСП, штриховая двухузельному приближению Бете, сплошная - четырехузельному приближению Бете. Показаны области существования неупорядоченной фазы (NO), зарядово-упорядоченной фазы (CO), сверхтекучей фазы (SF) и фазового расслоения (PS). 
тового Монте-Карло это уменьшение составляет $50 \%$. Для SF-фазы максимальная температура упорядочения при учете ближних корреляций также уменьшается, а диапазон концентраций увеличивается. Трикритическая точка смещается в сторону половинного заполнения вдоль линии CO-PS. В целом, учет ближних корреляций в рамках двух- и четырехузельного приближения Бете количественно приближает вид фазовой диаграммы к результатам, полученным методом квантового МонтеКарло [22], однако качественная особенность, связанная с наличием перехода $\mathrm{NO}-\mathrm{PS}$, в приближении Бете не воспроизводится: крайней точкой области PS в приближении Бете является трикритическая точка.

\section{4. Заключение}

Мы рассмотрели влияние учета ближних корреляций для двумерной модели локальных бозонов на квадратной решетке в рамках приближения Бете с использованием кластеров из двух и четырех узлов. Получены явные уравнения для критических температур зарядового и сверхтекучего упорядочения в указанных приближениях. Анализ полученных решений для различных отношений параметра межцентровых заряд-зарядовых корреляций и интеграла переноса $V / t$ показывает качественные отличия концентрационных зависимостей критических температур по сравнению с ПСП. Для температур зарядового упорядочения при учете корреляций возникает критическое значение концентрации бозонов, ограничивающее область существования решений СО-типа. Для сверхтекучего упорядочения происходит редукция критической температуры вплоть до нулевых значений при половинном заполнении. Также построена фазовая диаграмма модели локальных бозонов, полученная в двухи четырехузельном приближении Бете при $V / t=3$ с учетом расслоения фаз в рамках построения Максвелла. Показано, что учет ближних корреляций в приближении Бете количественно приближает вид фазовой диаграммы к результатам, полученным методом квантового МонтеКарло [22].

\section{Финансирование работы}

Работа выполнена при поддержке Программы 211 Правительства Российской Федерации, соглашение № 02.A03.21.0006, и проекта FEUZ-2020-0054 Министерства образования и науки Российской Федерации.

\section{Конфликт интересов}

Авторы заявляют, что у них нет конфликта интересов.

\section{Список литературы}

[1] T. Matsubara, H. Matsuda. Prog. Theor. Phys. 16, 569 (1956).

[2] H.A. Gersch, G.C. Knollman. Phys. Rev. 129, 959 (1963).
[3] R. Micnas, J. Ranninger, S. Robaszkiewicz. Rev. Mod. Phys. 62, 113 (1990).

[4] N.H. Lindner, A. Auerbach. Phys. Rev. B 81, 054512 (2010).

[5] O. Dutta, M. Gajda, Hauke, M. Lewenstein, D.-S. Lühmann, B.A. Malomed, T. Sowin'ski, J. Zakrzewski. Rep. Prog. Phys. 78, 066001 (2015).

[6] D. Abramavicius, A. Nemeth, F. Milota, J. Sperling, S. Mukamel, H.F. Kauffmann. Phys. Rev. Lett. 108, 067401 (2012).

[7] T. Giamarchi, C. Rüegg, O. Tchernyshyov. Nature Phys. 4, 198 (2008).

[8] J. Chang, E. Blackburn, A.T. Holmes, N.B. Christensen, J. Larsen, J. Mesot, R. Liang, D.A. Bonn, W.N. Hardy, A. Watenphul, M.V. Zimmermann, E.M. Forgan, S.M. Hayden. Nature Phys. 8, 871 (2012).

[9] G. Ghiringhelli, M. Le Tacon, M. Minola, S. BlancoCanosa, C. Mazzoli, N.B. Brookes, G.M. De Luca, A. Frano, D.G. Hawthorn, F. He, T. Loew, M.M. Sala, D.C. Peets, M. Salluzzo, E. Schierle, R. Sutarto, G.A. Sawatzky, E. Weschke, B. Keimer, L. Braicovich. Science 337, 821 (2012).

[10] E.H. da Silva Neto, Aynajian, A. Frano, R. Comin, E. Schierle, E. Weschke, A. Gyenis, J. Wen, J. Schneeloch, Z. Xu, S. Ono, G. Gu, M. Le Tacon, A. Yazdani. Science 343, 393 (2014).

[11] S. Robaszkiewicz, R. Micnas, K.A. Chao. Phys. Rev. B. 23, 1447 (1981).

[12] S. Wessel, M. Troyer. Phys. Rev. Lett. 95, 127205 (2005).

[13] D. Heidarian, K. Damle. Phys. Rev. Lett. 95, 127206 (2005).

[14] R.G. Melko, A. Paramekanti, A.A. Burkov, A. Vishwanath, D.N. Sheng, L. Balents. Phys. Rev. Lett. 95, 127207 (2005).

[15] R. Suzuki, A. Koga. J. Phys. Soc. Jpn 83, 064003 (2014).

[16] L. Dang, M. Boninsegni, L. Pollet. Phys. Rev. B 78, 132512 (2008).

[17] K.K. Ng. Y.C. Chen. J. Phys.: Condens. Matter 22, 185601 (2010).

[18] B. Capogrosso-Sansone, C. Trefzger, M. Lewenstein, P. Zoller, G. Pupillo. Phys. Rev. Lett. 104, 125301 (2010).

[19] S. Kar, S. Yarlagadda. Ann. Phys. (N.Y). 375, 322 (2016).

[20] G.G. Batrouni, R.T. Scalettar. Phys. Rev. Lett. 84, 1599 (2000)

[21] F. Hebert, G.G. Batrouni, R.T. Scalettar, G. Schmid, M. Troyer, A. Dorneich. Phys. Rev. B 65, 014513 (2001).

[22] G. Schmid, S. Todo, M. Troyer, A. Dorneich. Phys. Rev. Lett. 88, 167208 (2002).

[23] G. Murthy, D. Arovas, A. Auerbach. Phys. Rev. B 55, 3104 (1997).

[24] C. Pich, E. Frey. Phys. Rev. B 57, 13712 (1998).

[25] K. Bernardet, G.G. Batrouni, J.-L. Meunier, G. Schmid, M. Troyer, A. Dorneich. Phys. Rev. B 65, 104519 (2002).

[26] N.G. Zhang, C.L. Henley. Phys. Rev. B 68, 014506 (2003).

[27] E. Altman, W. Hofstetter, E. Demler, M.D. Lukin. New J. Phys. 5, 113 (2003).

[28] R. Micnas. Phys. Rev. B 76, 184507 (2007).

[29] T.N. Antsygina, M.I. Poltavskaya, I.I. Poltavsky, K.A. Chishko. Phys. Rev. B 80, 174511 (2009).

[30] K. Kapcia, S. Robaszkiewicz, R. Micnas. J. Phys. Condens. Matter. 24, 215601 (2012).

[31] K. Kapcia. J. Supercond. Nov. Magn. 27, 913 (2014).

[32] K.J. Kapcia, S. Murawski, W. Kłobus, S. Robaszkiewicz. Physica A 437, 218 (2015).

Редактор Т.Н. Василевская 